\title{
FROM METAPHOR TO ACTION EMBODIED LANGUAGE COGNITION
}

\author{
Pilar CASADO
}

The traditional theories that tried to explain how brain activity leads to cognition used the computer metaphor to describe the way the brain processes information codifying it into symbols that can combine following a finite set of rules. However, recent studies with updated paradigms that used neuroimaging techniques show that the brain activity registered when we observed a brain involved in some cognitive task are the same we would observe if that brain was immersed in perceptual, motor or emotional processing of the information handled for the task. In this article, we review the latest contributions to embodied cognition theories that provide a new approach to human cognition.

Keywords: symbols, embodiment, language, sensorimotor system, emotion.

The human species managed to adapt to almost every environment in the world we inhabit. We can list many qualities that might have contributed to this enormous adaptation ability, such as the high technological level reached by the species, brain plasticity or even social capabilities. However, we could sum all of them up in the singularity of the cognitive system, understood in the broadest sense, as a general processing system for the information provided by the environment, with the goal of solving problems derived from adapting to it.

Already in the first approaches to cognitive science, this singularity is explained from a computational perspective, according to which input information is processed thanks to a number of operations, based in a finite (larger or smaller) set of rules, on «symbolic» representations of the world. This is an abstract way to understand human cognition, because it establishes that the sensory modality (auditory, visual...) of the information input does not matter; ultimately, it will always be transformed into symbols; that is to say, it is independent from modality. It also assumes that it relies on computer-encapsulated modules, selfsufficient and isolated, which are responsible for different processing types (De Vega, Glenberg, \& Graesser, 2008).

Nonetheless, the latest research carried out in different fields, such as developmental psychology or neuroscience,

revealed that cognition is based in a dynamic relationship between the body and the environment, in how the organism interacts with the environment, rather than in an abstract process. In this new line of thinking, symbols are replaced with sensorimotor schemes that simulate perceptual experiences from our environment. Embodied cognition theories state that the joint action of the body and the environment supports cognitive processing

(Urrutia \& De Vega, 2011).

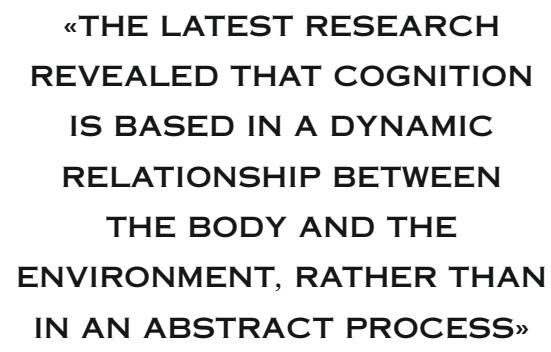

«THE LATEST RESEARCH REVEALED THAT COGNITION IS BASED IN A DYNAMIC RELATIONSHIP BETWEEN THE BODY AND THE ENVIRONMENT, RATHER THAN IN AN ABSTRACT PROCESS"

\section{LANGUAGE, ACTION AND PERCEPTION}

The relationship between language and the body is one of the most studied cognitive processes. This provided a number of significant findings supporting embodiment theories.

Debate over the symbolic and the embodiment stances regarding the understanding of the linguistic meaning is exemplified in the way it is produced. From a symbolic point of view, the meaning of words comes from symbolic associations with other words, while the embodiment perspective rejects the possibility that those associations exist and states that meaning needs to be constantly anchored to the real world.

This last approach has been endorsed by experiments with neuroimaging techniques that allowed to study variations in brain activity when specimens perform 


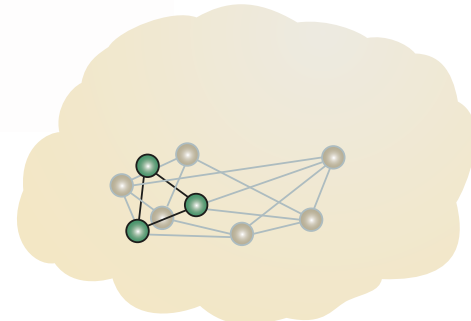

Face-related word

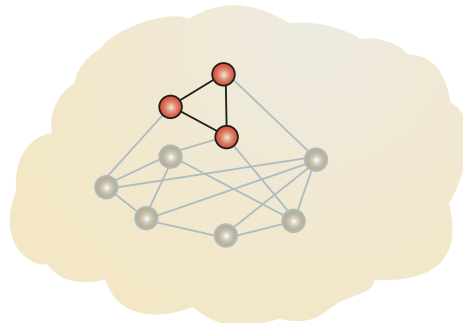

Arm-related word

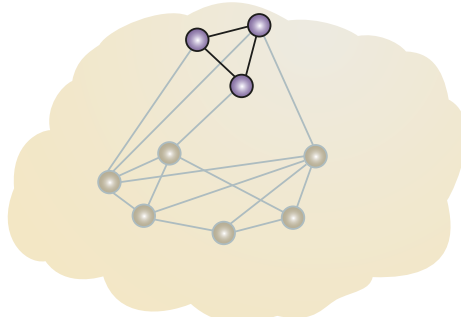

Leg-related word

Figure 1. Neural representation as functional networks of action words. The semantic circuits of words connected to actions related to movements of the face (green), arms (red) and legs (purple) involve neurons located in the corresponding somatotopic regions of motor and premotor areas.

Adapted from Pulvermüller and Fadiga (2010).

a task. The pioneering works by Pulvermüller stated that words are represented neurally by functional networks whose cortical distribution is determined by its meaning. If we compare words whose meaning is based essentially on sensory features (animals, for instance) with those whose meaning is more related to motor experiences (for example, tools), we will observe how different these networks are. While both word types activate areas related to linguistic processing (located around the Sylvian fissure), word fields related to action are completed with the activation of motor areas located in the frontal lobe, and sensory word fields activate posterior sensory areas (Pulvermüller \& Fadiga, 2010). In addition, comparing verbs (the main action words) involving different parts of the body shows that the activated area of the motor cortex is, specifically, the one involved in controlling the movement of the body parts related to those verbs (Pulvermüller, 2013) (Figure 1).

The embodiment approach enables also the study of the relationship between language and motor processes at a sentence level. The ACE paradigm (action-sentence compatibility effect) is useful to explore the existing relationship between comprehension of action sentences and motor mechanisms. In experiments where this paradigm is used, participants are asked to judge the coherence of sentences describing movements «from them» or «to them» $(« \mathrm{Liz}$ told you the story» vs. «You told Liz the story»), pushing or pulling a lever to issue their response. If it is true that understanding these sentences (in which an action is described) involves the co-activation of motor areas in the brain, a simultaneous movement «compatible» with what the sentence describes will make the required linguistic task easier for participants.

The results of this type of experiments show that reading times are significantly lower when the response and the sentence are compatible; that is to say, when the sentence described an action directed to the participants and they had to answer moving the lever «towards» them (also for sentences describing actions from the participants and responses involving pushing the lever away) (Glenberg \& Kaschak, 2002).

In the search for neural correlates for this compatibility effect, neuroimaging techniques have also been used; more specifically, the N400 component of eventrelated potential has been studied. This component constitutes a marker for semantic processing and appears when electrical brain activity is detected (thanks to electroencephalogram records) whenever semantic incongruity exists. Data reveal that during the processing of a sentence involving an action that is incompatible with the movement required of the participant, a semantic inconsistency occurs, reflected in the appearance of an N400 component (Figure 2). This suggests that the interaction between linguistic processing and motor processes observed with the ACE paradigm involves the existence of neural resources shared between the comprehension of action sentences and motor processes (Santana \& De Vega, 2013).

\section{LANGUAGE AND EMOTIONS}

As we said, according to embodiment theory, cognition is based on a dynamic relationship between the body and the environment. Until now, we have described how sensorimotor experiences intertwine with language, but the experience obtained thanks to the emotional system can also influence the ability to understand language.

In this sense, several recent studies have explored the existing relationship between the facial expression of emotion and processing sentences with emotional content. In one of the most salient ones, the researchers 
manipulated the state of the muscles in the participants' faces (Figure 3), asking them to hold a stick with their mouths horizontally (which made them simulate a smile) or vertically (which prevented them from smiling). At the same time, they were given sentences with positive emotional words, «compatible» with the horizontal position of the stick; or negative emotional words, «compatible» with the vertical position of the stick. The authors found that reading time for positive emotional sentences was shorter when the participants «smiled» than when the stick prevented them from smiling; and vice versa, when the sentence content was negative. This seems to reflect a compatibility effect (similar to the one seen in the previous section) between sentence and emotional expression. When the emotional content of the sentences coincides with the facial expression of the person reading it, comprehension is improved (Havas, Glenberg, \& Rinck, 2007).

In a later study, the same authors found that people who had undergone botox treatments found it more difficult to express several emotions (for instance, those that involved frowning, such as sadness). This noticeably affected their ability to understand the emotions of others, and even to understand sentences describing emotions. Specifically, reading times for sentences with sad content were significantly longer. The authors argue that, not being able to facially express an emotion makes it more difficult to understand that emotion in a sentence (Havas, Glenberg, Gutowski, Lucarelli, \& Davidson, 2010).

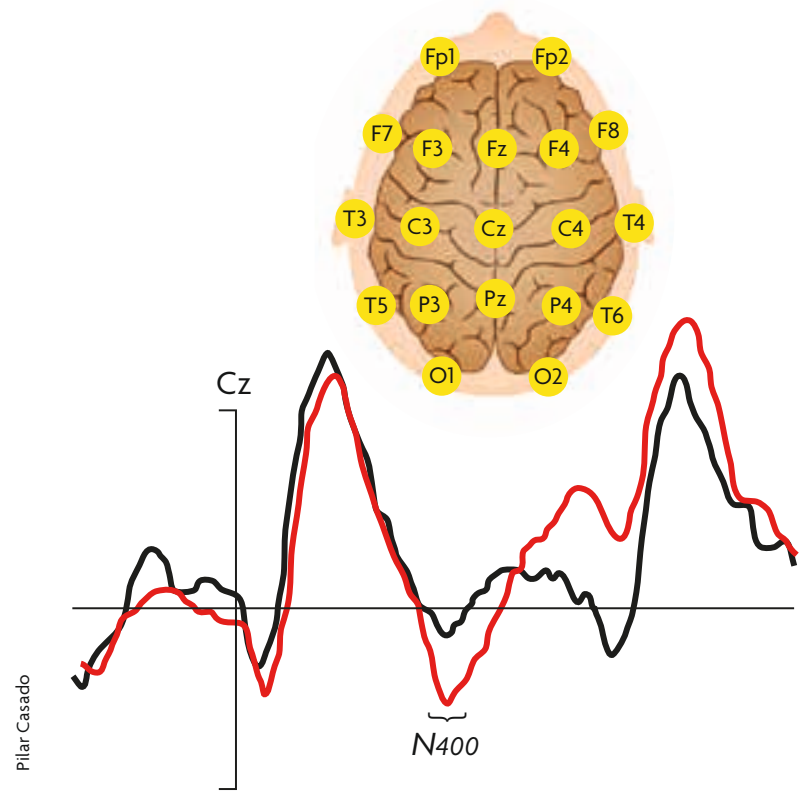

These studies support the hypothesis that the way the body experiences emotions (for example, through facial expression) affects cognitive processing related to them.

On the other hand, it is also interesting to study how language creates emotional states that influence cognitive processing. We have daily examples of how what others say affects us (waiters who receive higher tips when they deliver a joke with the bill...), but sports is the field were expressions of encouragement or discouragement are more emphasised in order to affect the performance of sportspeople. In our laboratory, we wanted to carry out an experiment to test whether expressions of encouragement or discouragement affect sports or not.

To this end, we designed an experiment in which participants had to perform a visuospatial attention task while they listened to encouraging or discouraging expressions. The electroencephalogram revealed the appearance of components from the requested task (P1, N1, SN and SP), but with modifications induced by the encouragement expressions. Our data showed that these expressions were able to modify the usual work pattern of the visual system, apparently leading the dorsal stream to process a feature that is usually processed by the ventral stream, like shape (Figure 4). Thus, we can confirm that linguistic expressions of encouragement, such as the ones usually present in sports and in many other contexts and situations, are efficient to exert emotional reactions and noticeable and measurable effects in cognition (MartínLoeches, Sel, Casado, Jiménez, \& Castellanos, 2009).
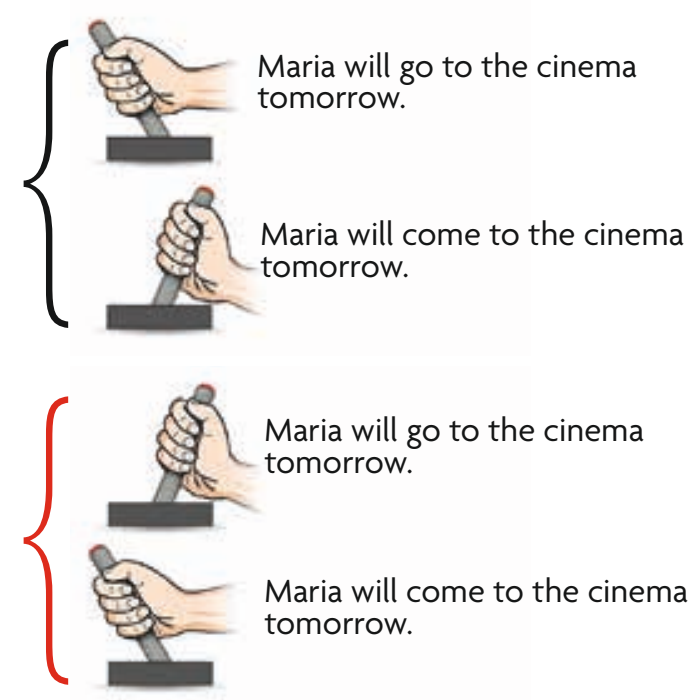

Figure 2. Using the ACE (action-sentence compatibility effect) in experiments with event-related potentials allows to measure incompatibility effects between processing an action sentence and performing a motor task that is incompatible with what the sentence expressed. The main result of these experiments is the appearance of the N400 component, consisting on a negative wave with centroparietal topography (maximum at the $\mathrm{Cz}$ electrode; the location of the electrode on the scalp according to the International System 10-20 is shown in the diagram at the top of the figure) and a latency of around $400 \mathrm{~ms}$; it constitutes an index of semantic inconsistency. 


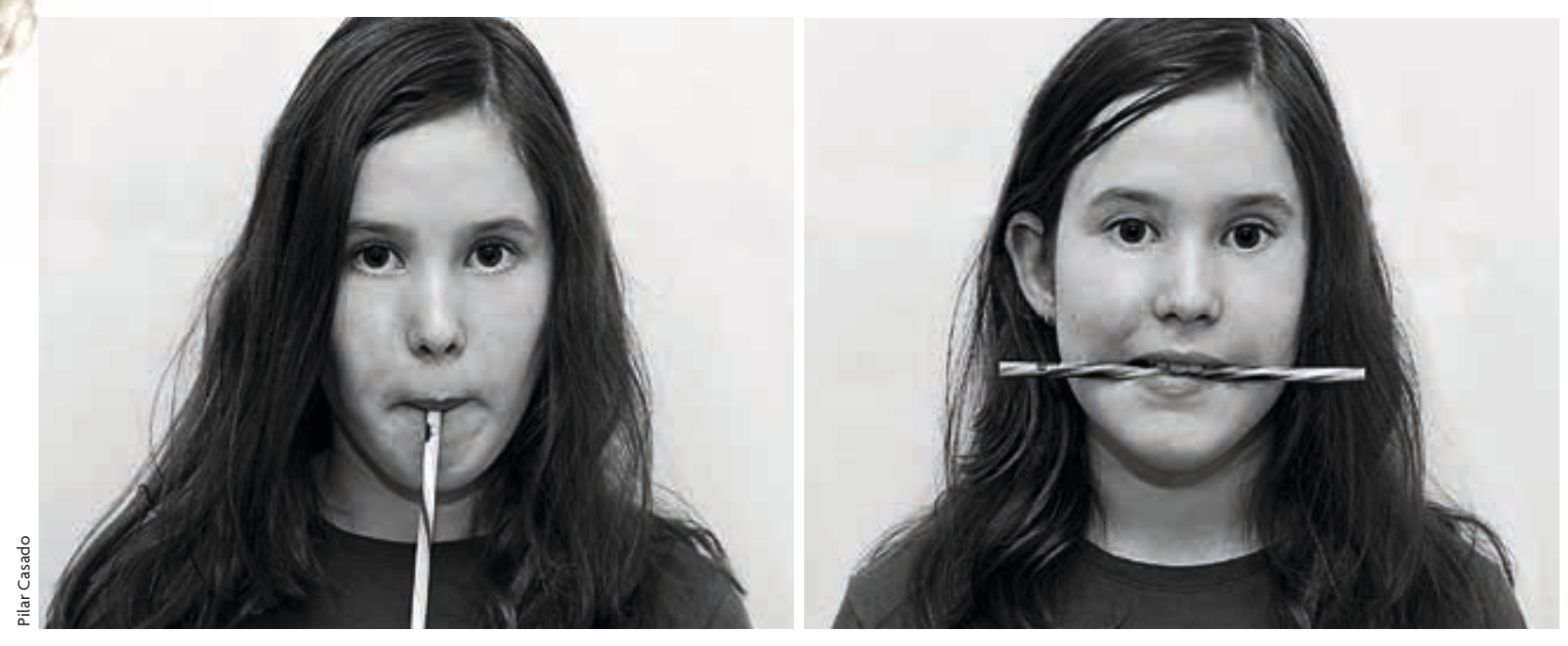

Figure 3. The facial expression of emotions can interfere with cognitive processing, even when the expression is artificial, as shown in the pictures in which holding a pencil in the mouth simulates a smile or a sad face with the corners of the mouth pointing down.

\section{EVOLUTION OF LANGUAGE}

Studies on the process of encephalisation of the Homo genus have highlighted the fact that the brain of our species is rather peculiar when compared to the rest of species in the genus. Among its differential characteristics, we can highlight the remarkable increase of some parts of the frontal lobe, the existence of differences, both functional and anatomical, between the two brain hemispheres and, especially, their globular shape, due to an expansion in the temporal and parietal lobes in our brain. The areas that show relevant increases were the motor and sensory areas, as well as areas of heteromodal association, where information from different sensory modes is integrated with motor and emotional information. These areas of the brain are also involved in different aspects of human language processing (Martín-Loeches, Casado, \& Sel, 2008).

In this sense, some authors have suggested that language might have emerged thanks to these connections between temporoparietal and prefrontal areas, which constitute also the neural substrate where the working memory is located in the brains of current humans and other primates. According to this, language would have evolved from a primitive system responsible for the active maintenance of sensorimotor information during the execution of some cognitive tasks. This rudimentary working memory system (and the brain areas responsible for it) would have evolved gradually, leading to the appearance of a conceptual system (and its phonological correlate) and a syntax, a system responsible for the manipulation of on-line information, dependent on the meaning of concepts (Aboitiz, García, Bosman, \& Brunetti, 2006).

These findings contribute to the debate in the scientific community between gradualism and saltationism in the evolution of human language. Some gradualist authors defend the idea that the dynamics of the process were slow and gradual, and were based on pre-existing structures and capabilities; for saltationists, the unique characteristics of our communicative system can only have emerged from an exceptional mutation, disconnected from previous structures and functions.

\section{EMBODIED METAPHORS}

If there is a paradigm of the relationship between body and language in everyday language, it is metaphors. The book Metaphors we live by, published by Lakoff and Johnson in 1998, was the first approach to a theory to explain how the way we represent (and self-represent) our body organises the human conceptual system.

One of the most common metaphors, present in many different cultures, uses spatial orientation (for example, up and down) to structure more abstract concepts, leading to expressions related to spatial orientation that use our own body as reference (north is up, so we "go up to the mountains"), or even metaphors with more emotional or moral content. Thus, "good» is up and «bad» is down (as in the JudeoChristian representation of heaven and hell), and we organise our emotions in a vertical scale. Consequently, good times «lift our spirits» and bad news make us «fall into depression".

These examples highlight how physical experience, both perceptual and motor, is inextricably linked to the way we organise our conceptual system; that is, the close relationship between body and language. 


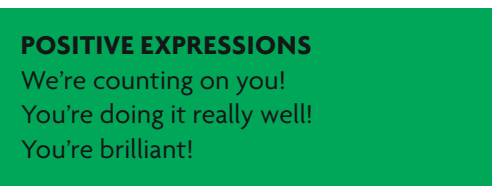

\section{NEUTRAL EXPRESSIONS}

Don't rub your eyes.

Set your eyes on this.

Relax.

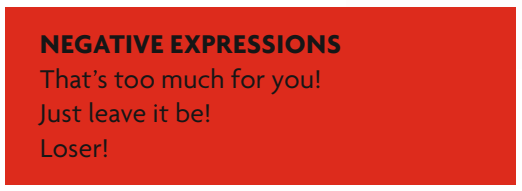

- Positive expressions

- Negative expressions

- Neutral expressions
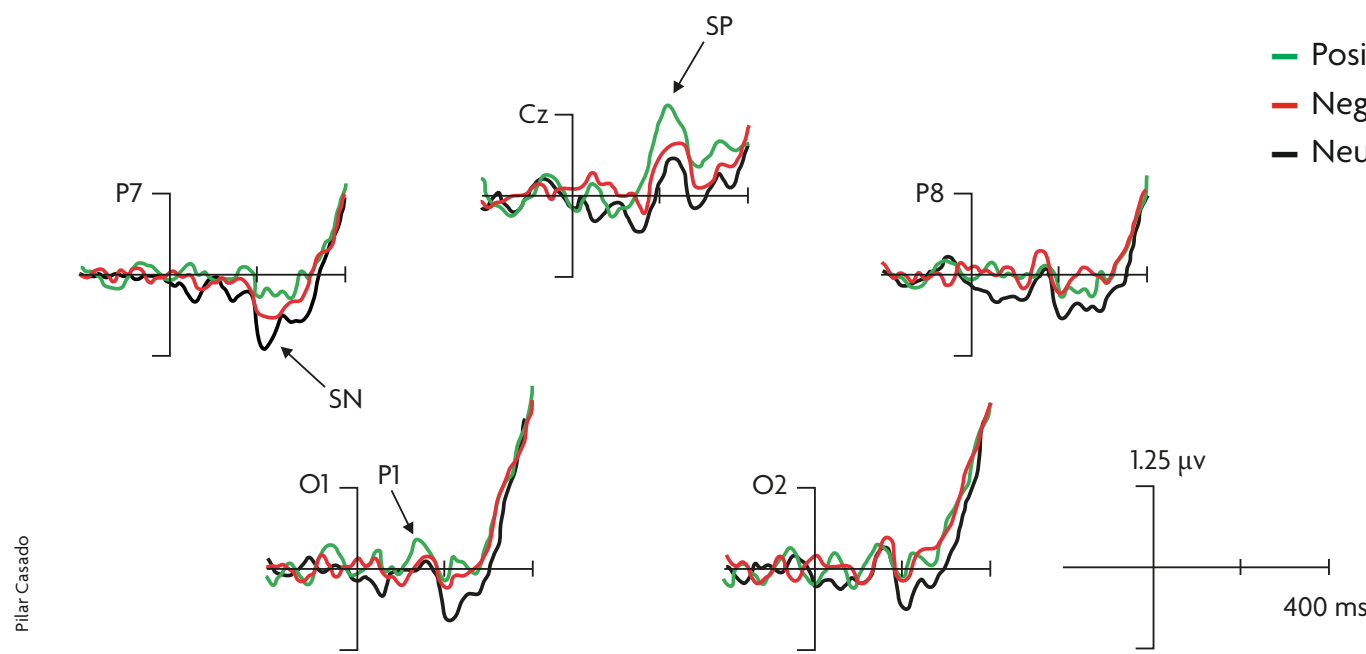

Figure 4. Using positive and encouraging expressions remarkably improves the results of visuospatial tasks, as proven in the modulation of the response to characteristics received from visual stimuli ( $\mathrm{Pl}$, SN and SP components). While the $\mathrm{Pl}$ component reflects the processing of spatial characteristics from the visual stimulus, the SN and SP components are related to the later processing of characteristics such as shape or colour.

The perspective of embodied cognition has made interesting contributions regarding the emergence and evolution of language. If, as we have defended throughout the text, language is not based on the existence of a specific and autonomous module, but rather shares neural mechanisms with other cognitive functions, this would support the hypothesis that language emerged from pre-existing neural structures and evolved through a gradual process.

The data show the embodied nature of language, in the sense that the neural systems involved in linguistic comprehension coincide with sensorimotor systems. The contributions of comparative anatomy also show how these systems evolved, and support the evolutionary theses about the emergence and gradual evolution of language. More specifically, the frontal-parietal system responsible for the active maintenance of sensorimotor information in primitive hominids was co-opted by language; that is to say, language is the result of a series of exaptations of brain mechanisms governing the relationship between the organism and its environment.

\section{REFERENCES}

Aboitiz, F., García, R., Bosman, C., \& Brunetti, E. (2006). Cortical memory mechanisms and language origins. Brain and Language, 98, 40-56. doi: 10.1016/j.bandl.2006.01.006

De Vega, M., Glenberg, A., \& Graesser, A. (Eds.). (2008). Symbols and embodiment: Debates on meaning and cognition. New York: Oxford University Press.
Glenberg, A. M., \& Kaschak, M. (2002). Grounding language in action. Psychonomic Bulletin \& Review, 9(3), 558-565. doi: 10.3758/ BF03196313

Havas, D. A., Glenberg, A. M., Gutowski, K. A., Lucarelli, M. J., \& Davidson, R. J. (2010). Cosmetic use of botulinum toxina affects processing of emotional language. Psychological Science, 21, 895-900. doi: $10.1177 / 0956797610374742$

Havas, D. A., Glenberg, A. M., \& Rinck, M. (2007). Emotion simulation during language comprehension. Psychonomic Bulletin \& Review, 14, 436-441. doi: 10.3758/BF03194085

Martín-Loeches, M., Casado, P., \& Sel, A. (2008). La evolución del cerebro en el género Homo: La neurobiología que nos hace diferentes. Revista de Neurología, 46, 731-741.

Martín-Loeches, M., Sel, A., Casado, P., Jiménez, L., \& Castellanos, L. (2009). Encouraging expressions affect the brain and alter visual attention. PLoS ONE, 4(6), e5920. doi: 10.1371/journal.pone.0005920

Pulvermüller, F. (2013). How neurons make meaning: Brain mechanisms for embodied and abstract-symbolic semantics. Trends in Cognitive Sciences, 17(9), 458-470

Pulvermüller, F., \& Fadiga, L. (2010). Active perception: Sensorimotor circuits as a cortical basis for language. Nature Reviews Neuroscience, 11, 351-360. doi: 10.1038/nrn2811

Santana, E., \& De Vega, M. (2013). An ERP study of motor compatibility effects in action language. Brain Research, 1526, 71-83. doi: 10.1016/j. brainres.2013.06.020

Urrutia, M., \& De Vega, M. (2011). Lenguaje y acción: Una revisión actual a las teorías corpóreas. Revista de Lingüística Teórica y Aplicada, 50(1), $39-67$.

Pilar Casado. Associate Professor of the Department of Psychobiology of the Complutense University of Madrid (Spain) and member of the Cognitive Neuroscience Unit, Center for Human Evolution and Behavior (UCM-ISCIII). Her research focuses on language processing, particularly on sentences and discourse, studied through event-related potentials and functional magnetic resonance. She is the author of many research papers on this topic. 